\title{
Larval dispersion along a straight coast with tidal currents: complex distribution patterns from a simple model
}

\author{
S. A. Richards, H. P. Possingham, B. J. Noye \\ Department of Applied Mathematics, University of Adelaide, Adelaide, South Australia 5005, Australia
}

\begin{abstract}
The majority of marine species have a complex life cycle where the adult phase is preceded by a pelagic larval phase. The dynamics of the more obvious adult phase may be strongly influenced by the distribution and abundance of larvae. Field experiments have been unable to give a complete picture of the spatial-temporal dynamics of the larval phase. This is due to the extremely small size of the individual larvae and the environment in which they live. Here we present a mathematical model of the dispersal of larvae into a region consisting of a straight coastline and a current dominated by tidal effects. Spawning is near the coast from a well-defined site the size of a small jetty or reef and the larvae have a relatively short pelagic lifetime. The model is based on the advection-diffusionmortality equation. Using a new analytic solution to the model, we examine the effect of processes such as the current structure, mortality, and the duration and rate at which larvae are released, on dispersal. The model is relatively simple but produces surprisingly complex patterns of dispersal. This has implications for attempts to produce more complex models of dispersal and the way in which field data of larval densities should be interpreted.
\end{abstract}

KEY WORDS: Complexity $\cdot$ Larvae $\cdot$ Mathematical model $\cdot$ Tidal current

\section{INTRODUCTION}

Many marine organisms have a complex life cycle in which the early stage is a pelagic larval form. Adults release their larvae into the surrounding water column where they are influenced by hydrodynamic processes like advection and eddy diffusion. This results in the dispersal of larvae away from their release site. Even though it has long been recognised that knowledge of the presettlement processes affecting larvae is important to understanding the population dynamics of the adult stage, recently there has been a stronger emphasis on this issue (Butler 1987, Menge \& Sutherland 1987, Roughgarden et al. 1988, Dight et al. 1990, Possingham \& Roughgarden 1990 , Sale 1990, Fairweather 1991, Havenhand 1991b, Minchinton \& Scheibling 1991, Gaines \& Bertress 1992, James \& Scandol 1992, Koehl et al. 1993). Previously, local populations were often modelled by considering local conditions only. It is now realised that the inclusion of conditions at a larger spatial scale is required to understand the large variations in recruitment observed in the field.

The larval phase is important when considering issues such as marine reserve design, environmental monitoring, and the impact of humans on populations. For species with a long pelagic larval phase in particular, changes in large-scale hydrological conditions can dramatically affect the dynamics of a local population. Knowledge of the processes involved in larval dispersal is important for understanding gene flow between spatially distinct communities, especially when the adult phase is sessile (Todd et al. 1988, Havenhand 1991a, Planes 1993). Where local extinctions of marine species occur, the extent of larval dispersal is critical for recolonisation

Very few critical experiments and measurements have been made on larval dispersal (Okubo 1980, Jackson \& Strathmann 1981, Barnes \& Mann 1991). This has been due to the difficulties in observing and collecting such small organisms in the field (Strathmann 1974, Fairweather 1991, Scandol \& James 1992). 
The purpose of this paper is to construct a mathematical model which tracks the movements of a large number of larvae when released from a well-defined region into a tidal current. The solution to the model is the spatially varying distribution and abundance of larvae within the larval cloud. This is the sort of information that field experiments have currently been unable to provide. The model is run under a variety of conditions, and the distribution patterns analysed to get a basic understanding of how certain processes influence and effect dispersal. In particular we assess the relative importance of currents, turbulence, pelagic lifetime, and the duration and rate of spawning. Measurements of larval densities within a spatially confined area can be performed by sampling the water column, or using settlement plates or traps (Minchinton \& Scheibling 1991). Our model is used to produce dispersal diagrams that predict the data that could be collected from these types of experiments and our results have significant implications for their interpretation.

The model in this paper is concerned with the early pelagic siage of the iife cycie and cuuld be used in a larger model which investigates recruitment and adult population dynamics. The model begins with the spawning of larvae from a release site the size of a jetty or small reef. Spawning is not instantaneous (although the model can treat this as a special case) but continues over a defined period of time. Released larvae are then mixed into the surrounding current. The result is to produce a cloud of larvae with a complicated internal density structure. The cloud is tracked over a few days and its distribution examined. We consider a specific current regime that is typical of those found in shallow gulfs and large estuaries (B. J. Noye pers. obs.). We assume that the tides create a current that oscillates in a direction parallel to the coast over a $12 \mathrm{~h}$ period (Barnes \& Mann 1991). We also consider the case when there is a steady along-shore drift acting in conjunction with the tidal oscillation and then compare the results. Even with these simple currents surprisingly complex patterns of larval dispersal arise and this has important. implications for the analysis of field data on larval abundance.

Larval movement is governed by the 2 -dimensional depth averaged advection-diffusion-mortality equation (ADME). Modelling the pelagic phase of an organism's life cycle by the ADME is not new (Jackson \& Strathmann 1981, Roughgarden et al. 1988, McGurk 1989, Possingham \& Roughgarden 1990, Hill 1990, 1991a, Hinckley et al. 1993). Generally, spatial and temporal scales considered in previous models have been large and smaller scale phenomena such as the oscillation component of the tidal current and the duration of spawning are neglected. For example, Hill
(1990) considered the dispersal of Norway lobster Nephrops norvegicus larvae in the western Irish Sea. The release site was $100 \mathrm{~km}$ by $100 \mathrm{~km}$ and the larvae were allowed to drift for a period of $50 \mathrm{~d}$ towards a recruitment site $300 \mathrm{~km}$ downstream. As mentioned above, in our model the release site is relatively small and larval drift is tracked only for a few days and remains relatively close to the coast. We show that the smaller-scale effects must be included in our model equations as they have a significant effect on the patterns of dispersal. Actual settlement is not incorporated into the model equations, but with a knowledge of the precompetent period, and the competent period of the particular species of interest, the model can be used to show the extent of coastline where larvae are competent to settle.

The structure of this paper is as follows. First we give the governing equation for the mathematical model and explain in which situations it is appropriate. We then examine and justify the parameters used in the model. The numerical results are then given and briefly discussed. In conclusion we examine the implications of the model.

\section{DERIVATIONS AND ASSUMPTIONS OF THE MODEL}

For many species of marine organisms the dispersal of larvae is largely controlled by horizontal currents (De Wolf 1973, Hannan 1984, Butman 1987, Walters 1992). Although some species of larvae have the ability to swim horizontally, the period when active swimming can be sustained, and the speed attained, is usually small when compared with the surrounding water movement. We assume that larvae act as if they are passive particles. For some species, development during the pelagic phase leads to the grouping of individuals (Power 1984, McGurk 1989). As we ignore horizontal swimming and schooling of larvae in our model, the results it produces may be relevant only for the early part of the pelagic phase for some species.

Diurnal vertical migration has been observed for some species of larvae. Typically, these larvae congregate in the surface waters during the night and recede into the depths during the day. However, this is not always the case and some species prefer to be at the surface during the day and near the bottom at night (Barnes \& Mann 1991). Our model is 2-dimensional and treats all variables as depth-averaged values so our results specifically apply if the larvae behave in 1 of 2 ways. Firstly, the larvae are neutrally buoyant and well mixed (evenly distributed) throughout the water column from the surface to the bottom. In this case the parameters used in the model such as the horizontal 
current velocities and the horizontal dispersion coefficients are depth averaged values. Alternatively, larvae maintain themselves within a bounded region in the vertical and they are evenly distributed within this region. For example, the larvae always remain within the first few metres of the surface (Johnson \& Hess 1990). Here, current and diffusion values represent averages over the region where the larvae reside.

The ADME generally used to model the depth-averaged dispersal of a passive particle in a turbulent fluid is given by

$$
\begin{aligned}
\frac{\partial}{\partial t}(h C)+ & \frac{\partial}{\partial x}(h U C)+\frac{\partial}{\partial y}(h V C)= \\
& \frac{\partial}{\partial x}\left(K_{x x} \frac{\partial}{\partial x}(h C)\right)+\frac{\partial}{\partial x}\left(K_{x y} \frac{\partial}{\partial y}(h C)\right)+ \\
& \frac{\partial}{\partial y}\left(K_{y x} \frac{\partial}{\partial x}(h C)\right)+\frac{\partial}{\partial y}\left(K_{y y} \frac{\partial}{\partial y}(h C)\right)-M h C
\end{aligned}
$$

where $x$ and $y$ are horizontal Cartesian coordinates; $t$ is time; $h(x, y, t)$ is water depth; $C(x, y, t)$ is depthaveraged concentration of larvae per unit volume; $U(x, y, t)$ and $V(x, y, t)$ are depth-averaged water velocities in the $x$ and $y$ directions respectively; $K_{x x}(x, y, t)$, $K_{x y}(x, y, t), K_{y x}(x, y, t)$ and $K_{y y}(x, y, t)$ are depth-averaged components of the diffusion tensor $K_{;}$and $M(x, y, t)$ is the depth averaged coefficient for mortality. Uppercase notation is used to indicate variables that are depth-averaged. Finding an analytic solution for Eq. (1) is possible only if some assumptions are made on the boundary conditions and the functional forms of the parameters.

We consider dispersal within a region bounded on 1 side by a long straight coastline (or estuary bank) that coincides with the $x$-axis (see Fig. 1). We assume that the effect of the tide is to produce a current that is parallel to the coastline such that at any instant in time it has uniform strength and direction everywhere. Thus the depth-averaged across-shore current velocity $V(x, y, t)$ is set to zero and the depth-averaged alongshore current velocity becomes a function of time only, i.e. $U=U(t)$.

The spreading of the larval cloud is due to the terms containing the components of the dispersion tensor $K$. These components are used to represent turbulence caused by the existence of eddies (erratic circular motions of water) which distort and mix the larval cloud. The resulting effect of an eddy on the larval cloud is dependent on scale. If the size of the eddy is much larger than that of the larval cloud, then the cloud will tend to be advected as a whole following the motion of the eddy, whereas if the eddy is smaller than the cloud, it will tend to mix and break up the cloud, resulting in an overall increase in size of the cloud (Bowden 1964, Okubo 1980). Intuitively, the longer a cloud is exposed to eddies, the larger it will become and hence eddies which initially tended to advect the cloud will later aid in the process of breaking it up. Thus, if there exists eddies at all spatial scales, then the rate at which the larval cloud spreads should increase with time. Turbulence theory predicts that with a point source release the variance of a cloud will follow the third power of time, $t$. Experimental results given by Okubo (1971) found that a best line of fit for the data gave a power of 2.34. Okubo (1971) also related an apparent diffusivity, $K_{a}$, with the scale of the cloud, 1 . Theory predicts that $K_{a}$ should follow the $4 / 3$ power law, which is slightly higher than the 1.15 observed from data (Okubo 1971).

It should be noted that the diffusion tensor used in Eq. (1) represents a statistical average of the effects of diffusion. In reality eddies will distort a larval cloud resulting in it having a very complicated boundary and internal concentration structure (Fischer et al. 1979). This irregularity can not be resolved by Eq. (1); what is obtained is an average (not typical) concentration profile of the cloud, hence the model gives a smoother distribution of larvae than would be observed in the field.

We make the assumption that diffusion is composed of longitudinal and transverse components only (Holly \& Usseglio 1984). As flow is in the $x$-direction only, we set the diffusion tensor components $K_{x y}=K_{y x}=0$. Like the advection term $U$, we assume spatial independence of the 2 remaining diffusion components, i.e. $K_{x x}=K_{x x}(t)$ and $K_{y y}=K_{y y}(t)$. This means that at any instant in time the turbulence contributing to diffusion of the larval cloud in the $x$-direction is the same throughout the region and similarly for diffusion in the $y$-direction. In reality the presence of the coastline and the depth of the water may influence the rate of diffusion.

For this model larval mortality, $M$, is assumed to be due only to predation and starvation. A more complicated model may also include mortality effects due to inhospitable environmental conditions such as extremes in salinity and temperature. Again, spatial dependence is ignored and so $M=M(t)$ and larvae are influenced equally throughout the entire region but the rate of mortality may change with larval age.

In order to simplify Eq. (1) to a stage where an analytic solution may be found we assume that the water depth is independent of $x$ and $t$, so $h=h(y)$. Thus, tidal sea-surface elevations are considered to be negligible when compared with the mean depth of the region being modelled and that there is uniformity in the seabottom bathymetry along the coast.

The requirement that all of the parameters to the model be spatially independent is a limitation of the model. Thus, the results given in this paper may not be 
applicable to the situation when environmental conditions, such as eddy turbulence, larval mortality, etc., vary considerably throughout the region where dispersal takes place. We feel that spatial independence is a reasonable first approximation when modelling dispersal over the relatively small range of tens of kilometres.

With the above assumptions the ADME is simplified to,

$$
\frac{\partial S}{\partial t}+U \frac{\partial S}{\partial x}=K_{x x} \frac{\partial^{2} S}{\partial x^{2}}+K_{y y} \frac{\partial^{2} S}{\partial y^{2}}-M S
$$

where $S=S(x, y, t)=h(y) C(x, y, t) ; U=U(t) ; K_{x x}=K_{x x}(t)$; $K_{y \gamma}=K_{y y}(t)$ and $M=M(t)$. The unknown variable $S$ is the depth-integrated concentration which by definition is the product of the depth-averaged concentration per unit volume and the water depth. This equation is similar to the one used previously (Possingham \& Roughgarden 1990, Hill 1990, 1991a) but is more general as the advection, diffusion and mortality terms may now be time dependent.

\section{PARAMETER ESTIMATION}

One of the most difficult aspects of modelling is estimating realistic values for parameters and functions for relationships (Hofmann 1993). In Eq. (2) we have 4 parameters that require a functional form. They are the along-shore velocity profile $U(t)$, the diffusion components $K_{x x}(t)$ and $K_{y y}(t)$, and the mortality rate $M(t)$. We also need to define the size and location of the release site, the rate at which larvae are released from this site, and the time that larvae spend in the water column before they can settle.

First, consider the region where the marine species releases its larvae. The release site might be a reef, mud flat or jetty. To simplify the analytic solution let the release site be rectangular in shape with centre at $\left(x_{R}, y_{R}\right)$ and with sides of length $2 A_{R}$ and $2 B_{R}$ (Fig. 1$)$. With all the examples given in this paper we set $\left(x_{R}, y_{R}\right)$

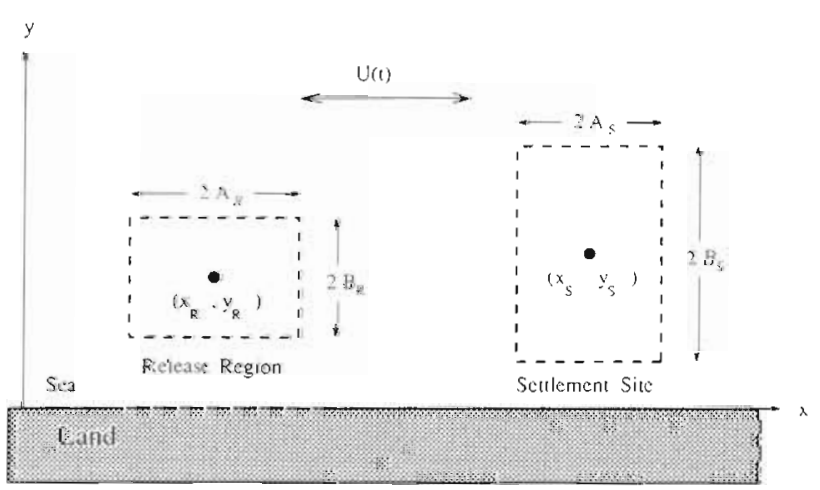

Fig. 1. The geometry of the solution domain
$=(0,200 \mathrm{~m}) ; A_{\mathrm{R}}=5 \mathrm{~m} ;$ and $B_{\mathrm{R}}=100 \mathrm{~m}$. This set of values is used to represent a small $200 \mathrm{~m}$ long jetty or reef projecting from the coast. We consider a potential settlement site having the same dimensions as the release site (i.e, another jetty or small reef) located $4 \mathrm{~km}$ downstream. The term downstream refers to the positive $x$ direction while upstream refers to the negative $x$ direction. If we use similar notation to define the dimensions and location of the settlement site as the release site then $\left(x_{S}, y_{S}\right)=(4000 \mathrm{~m}, 200 \mathrm{~m}), A_{\mathrm{S}}=5 \mathrm{~m}$ and $B_{\mathrm{S}}=100 \mathrm{~m}$.

Next we need to know the number of larvae, denoted $L$, that are spawned from the release site. This number depends on the size of the release site and the species being modelled. Let $L=1000000-$ in the following results, densities of larvae should be compared to this original release number. The duration of larval release, $T$, also needs to be specified along with a timedependent rate, $r(t)$, representing the fraction of total larvae released at time $t$. As $r(t)$ is a fractional rate of release then

$$
\int_{0}^{T} r(\dot{t}) \mathrm{d} \dot{t}=\hat{\imath}
$$

We include this rate to represent fluctuations in larval release that may occur. For example larvae may be more likely to be released during periods of darkness (Strathmann 1974) or when the surrounding current is near its peak strength. Note that the rate of release at any time during spawning is assumed to be equal throughout the release site. We can now calculate the rate of release of larvae per unit area within the release site, denoted $\phi$, by

$$
\phi(t)=\frac{L r(t)}{4 A_{\mathrm{R}} B_{\mathrm{R}}}
$$

In all examples given in this paper we assume that $r(t)$ is constant throughout the spawning period, $r(t)=$ $1 / T$ and $\phi(t)=\phi=L /\left(4 T A_{R} B_{R}\right)$. For example, with parameter values mentioned above if we release larvae continuously for $12 \mathrm{~h}$ ( 1 tidal cycle, see below) the rate of constant release is approximately 42 larvae $\mathrm{m}^{-2} \mathrm{~h}^{-1}$.

Tidal effects and an along-shore mean drift can be simulated by letting $U$ have the following form

$$
U(t)=u_{0}+\sum_{p=1}^{p} u_{p} \sin \left(\omega_{p} t+\varepsilon_{p}\right)
$$

where $P$ is the number of sinusoidal components making up the tidal motion, $t$ is time, $u_{0}$ is the mean alongshore velocity, and $u_{p}$ is the amplitude of the oscillatory component parallel to the shore having circular frequency $\omega_{p}$ and phase $\varepsilon_{p}$. We model a tide having a single dominant oscillatory component with period $12 \mathrm{~h}$, i.e. $P=1, \omega_{1}=\pi / 6$. The amplitude of this component, 


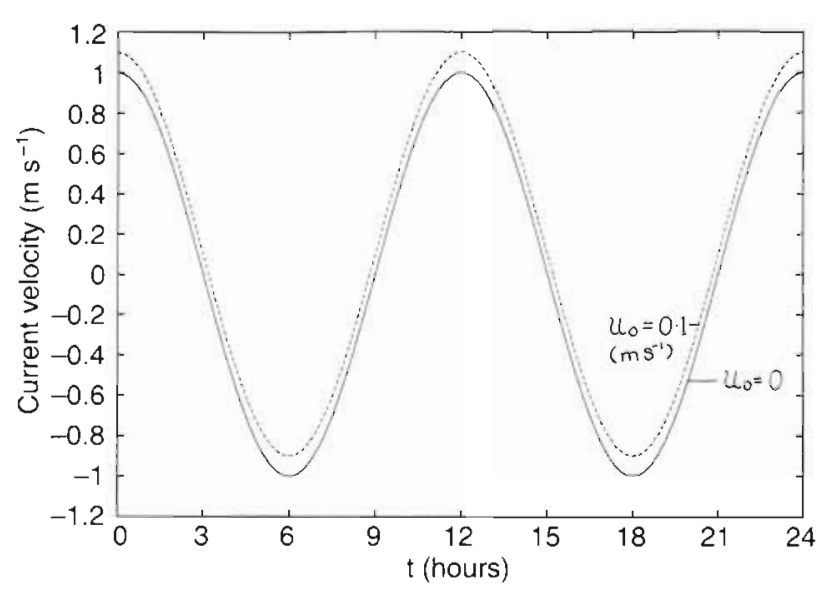

Fig. 2. The 2 current profiles, $U(t)$, used in the model simulations [i.e. Eqs. (6) \& (7)]

$u_{1}$, is set to $1 \mathrm{~m} \mathrm{~s}^{-1}$. This is typical of the current experienced in the gulfs of South Australia (Noye 1984). To see the effects of an along-shore drift on dispersal, 2 sets of model runs are given in the next section; the first assumes no along-shore drift, so $u_{0}=0$, and the other, a slow drift, $u_{0}=0.1 \mathrm{~m} \mathrm{~s}^{-1}$. For both cases we assume the velocity is initially at maximum strength in the downstream direction, i.e. $\varepsilon=\pi / 2$. The functional forms of these 2 velocity profiles is given in Eqs. (6) and (7). Graphs of the 2 velocity profiles are shown in Fig. 2. Although there appears to be little difference between these 2 velocity profiles the differences in the patterns of dispersal are quite pronounced.

$$
\begin{aligned}
& U(t)=3600 \sin \left(\frac{\pi t}{6}+\frac{\pi}{2}\right) \mathrm{m} \mathrm{h}^{-1} \\
& U(t)=360+3600 \sin \left(\frac{\pi t}{6}+\frac{\pi}{2}\right) \mathrm{m} \mathrm{h}^{-1}
\end{aligned}
$$

Finding functions to represent the typical effects of diffusion within coastal waters is difficult due to a lack of experimental data and the nature of the problem (Koehl et al. 1993). We have decided to use a range of values for the diffusion coefficients which hopefully bounds real values and it also allows us to see how changes in diffusion levels affect dispersal. The rate at which the larval cloud will diffuse is largely dependent on the cloud dimensions and the eddies within the region of the cloud. Initially, the size and shape of the cloud is primarily determined by the current regime and the duration of spawning. Consider the velocity profile of Eq. (6). If we ignore the effects of diffusion, the length in $x$ of the larval cloud will increase with time due to larvae being advected away from the release site by the current. The maximum length of the larvae cloud as a function of release time is plotted in Fig. 3. For release times greater than $12 \mathrm{~h}$ the maxi- mum length remains at approximately $13.75 \mathrm{~km}$. The length in $y$ of the cloud is fixed at $2 B_{\mathrm{R}}=200 \mathrm{~m}$, due to there being no water movement in the $y$-direction. Clearly the cloud extends a long way along the coast within a short period of time. A similar result occurs with the velocity profile of Eq. (7), although there is no upper limit to the length in $x$ that the cloud may attain. Okubo (1976) gives a functional form for the rate of diffusion which best fits data previously collected. This function, $K(r)$, assumes a point source release and is given by,

$$
K(I)=0.0680 r^{1.15}
$$

where $K$ and $r$ have units $\mathrm{cm}^{2} \mathrm{~s}^{-1}$ and $\mathrm{cm}$, respectively. From Eq. (8) we can find the rate of diffusion of a circular cloud of radius $r$. We assume the eddies that cause diffusion of a cloud of radius $r$ have themselves a radius less than or equal to $r$. Hence, if the size of the larval cloud is less than the size of the surrounding eddies, the rate of diffusion is determined by the size of the larval cloud but if the cloud is larger than the range of eddies, the rate of diffusion is bounded by the size of the largest eddies. The presence of the coastline reduces the maximum size of the eddies that can exist within the coastal waters.

Two base values for diffusion, $K$, are used in this paper. They are $K=1000 \mathrm{~m}^{2} \mathrm{~h}^{-1}$ and $K=10000 \mathrm{~m}^{2} \mathrm{~h}^{-1}$. Substituting these values into Eq. (8) and allowing for the difference in scale we can obtain a radius value that corresponds to the rate of diffusion. For $K=$ $1000 \mathrm{~m}^{2} \mathrm{~h}^{-1}, r \approx 102.3 \mathrm{~m}$ and for $K=10000 \mathrm{~m}^{2} \mathrm{~h}^{-1}, r \approx$ $757.4 \mathrm{~m}$. In our model we assume that the eddies

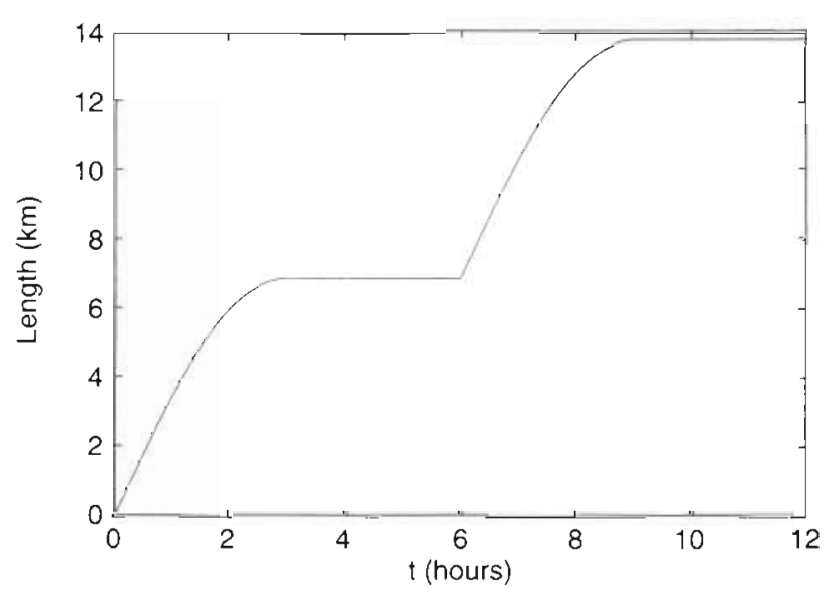

Fig. 3. Approximate length of the larval cloud when diffusion effects are ignored and larvae are released continuously for $12 \mathrm{~h}$ into the current regime given by Eq. (6). The release begins at $t=0$ when the flow is at its maximum strength in the downstream direction. This graph is derived by integrating Eq. (6) 
within the coastal waters are bounded in radial size by either approximately $100 \mathrm{~m}$ or $750 \mathrm{~m}$. Recalling the result given above on the initial size of the larval cloud, if we let spawning take place for more than a few hours the larval cloud will quickly attain a length that is much greater than that of the eddies. Thus it is reasonable to assume that the rate of diffusion is very near constant for all time. Note that if instead larvae were released instantaneously as a single point source, then a time dependent rate of diffusion based on the result given by Eq. (8) would be more appropriate.

Observations in the ocean have shown that a cloud of particles tends to be elongated in the direction of flow (Bowden 1964, Okubo 1980), commonly referred to as the 'shear effect'. This effect is due to the vertical diffusion of water mass within a sheared vertical velocity profile. Particles (or larvae) at differing depths are advected with different speeds resulting in an apparent increase in the horizontal diffusion of the particles in the direction of the current. The increased rate of diffusion is dependent on the rate of vertical mixing and the vertical velocity profile. Bowden (1964) quoted an increase in the rate of dîf̂rusion by a factor of 10 while Holly \& Usseglio (1984) suggested the increase factor to lie in the range of 20 to 30 . Here we assume that diffusion is influenced by shear effects so diffusion in the $x$-direction is 10 times greater than that in the $y$-direction i.e. $K_{x x}=10 K_{y y}$. Considering 2 base values of diffusion we run the model for both velocity profiles given by Eqs. (6) and (7) with the 2 pairs of diffusion parameters given in Table 1.

The rate at which larvae are lost, $M(t)$, is influenced by biotic and abiotic factors, i.e. food, predators, and temperature. Mortality rates of pelagic larvae are difficult to measure and are bound to be variable. Jackson \& Strathmann (1981) quote a range of figures from $0.034 \mathrm{~d}^{-1}$ for a crab of the genus Halicarcinus up to $0.65 \mathrm{~d}^{-1}$ for Calanus nauplii. Hinckley et al. (1993) performed a field study to estimate an age-dependent rate of mortality for walleye pollock larvae. They found that mortality rates were generally higher for young fish larvae and decreased with age and size. The maximum rate estimated from this study was $0.0575 \mathrm{~d}^{-1}$, which was low when compared with previous estimates for the same species. For all the results given in the next section we set the mortality rate to be constant at $0.02 \mathrm{~h}^{-1}\left(0.48 \mathrm{~d}^{-1}\right)$. At this rate approximately $38 \%$ of the larvae die per day.

In this paper we track the movement of larvae for only a few days as the important characteristics of dispersal produced by our model can be detected within this period of time. An example of a species that has a larval stage which spends several days in the plankton is the greenlip abalone (Shepherd et al. 1992).

\section{RESULTS}

Subject to the reflective boundary condition at the coastline, Eq. (2) can be solved to give an analytic solution, $S(x, y, t)$, provided the release of larvae is assumed to be instantaneous. To obtain a solution for the case when larvae are released continuously over a period of length $T$, we integrate the solution for the instantaneous release with respect to time over the release period. We solved the integral analytically using the simple Composite Simpson Rule (Mathews 1987, Conte \& de Boor 1988).

The number of larvae, $N(t)$, within the water column above a settlement site at a particular time can be calculated by integrating the depth integrated concentration, $S(x, y, t)$, over the settlement site i.e.

$$
N(t)=\int_{y_{S}-B_{S}}^{y_{S}+B_{S}} \int_{x_{S} A_{S}}^{x_{S}+A_{S}} S(x, y, t) \mathrm{d} x \mathrm{~d} y
$$

Like the function $S(x, y, t)$, an analytic solution for $N(t)$ can be found provided the release of larvae is assumed to be instantaneous, whereas numerical integration is required if larvae are released uver a period of time. The mathematical solutions to Eqs. (2) and (9) are given in the 'Appendix'.

The release of larvae into the current described by Eq. (6) is first considered. If we ignore the effects of eddy diffusion then an approximation to the size and location of the larval cloud can be made from the current velocity profile. For the case considered above, where larvae are released over a $12 \mathrm{~h}$ period, the approximate distribution of larvae along the coast is given in Fig. 4. At time $t=0$, larval release begins from the release site located at $x=0$. Initially the current is at its maximum strength and larvae are quickly moved downstream. For the next $3 \mathrm{~h}$ the current gradually

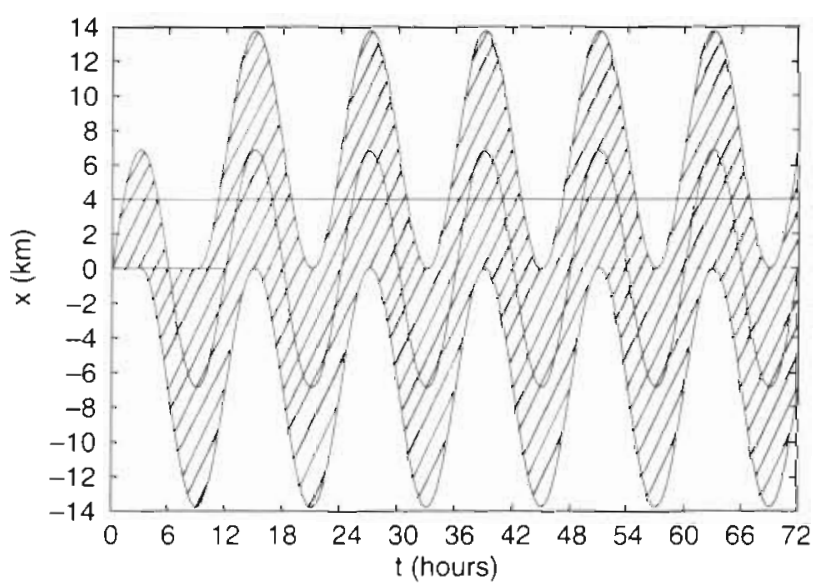

Fig. 4. Approximate along-shore distribution of larvae when released continuously for $12 \mathrm{~h}$ into the current regime given by Eq. (6) 
decreases in strength until eventually the water becomes still (slack water), by this time the larval cloud extends nearly $7 \mathrm{~km}$ downstream starting from the release site. The current then reverses direction and for the next $6 \mathrm{~h}$ larvae are advected upstream until another slack occurs. From the graph we can see that after $9 \mathrm{~h}$ of release the larval cloud extends nearly $14 \mathrm{~km}$ upstream from the release site. This is the maximum length obtained by the larval cloud (if we ignore diffusion processes) and the effect of the current after the 9th hour is to periodically advect the cloud up and down the coast about the central release site.

By ruling a line parallel to the $t$-axis which intersects the $x$-axis at the $4 \mathrm{~km}$ mark in Fig. 4, we can predict when larvae will be present at the potential settlement site. Observing where the straight line intersects the curves we can see that larvae should first appear approximately between the 1st and 5th hours. Larvae are next expected to appear between the 11 th and 19 th hours. Fig. 4 also shows that not all of the larval cloud actually passes the potential settlement site; in fact, a section at the upstream end of the cloud is never observed at the downstream site as the cloud drifts periodically up and down the coast with the current.

Fig. 5 shows the number of larvae, $N(t)$, at the potential settlement site for the 2 sets of eddy diffusion rates given in Table 1. Both graphs are similar in shape and agree with the predicted times when larvae should be present. It can be seen that the number of larvae over the settlement site, $N(t)$, fluctuates a lot initially, but then settles down to become more smooth and periodic. In order to understand how the large spikes and sharp jumps in concentration are formed, we need to consider what is happening at the time of release.

The density of larvae at the point of release is dependent on the rate of release and the speed of surrounding water. Here, larvae are released at a constant rate throughout the spawning period and so fluctuations in the concentration profile can be determined from the current. At times of slack water during the release period (i.e. $t=3$ and $9 \mathrm{~h}$ ) we would expect the concentration of larvae at the release site to be higher than at any other time during the release. This is because the volume of water into which the same number of larvae are released is smaller at slack periods than at times when the current is faster. Thus high larval concentrations are produced at the release site at both the $3 \mathrm{rd}$ and 9th hours. These high concentrations are then advected by the current along the outer curves given in Fig. 4. Thus, after $9 \mathrm{~h}$ of release 2 peaks in concentration are located at both ends of the cloud. It is interesting to note that a jump in concentration will occur $6 \mathrm{~h}$ after release. This jump occurs because the larvae released at $t=0$ return back to the release site after $6 \mathrm{~h}$ of drifting with the tide where they then mix with
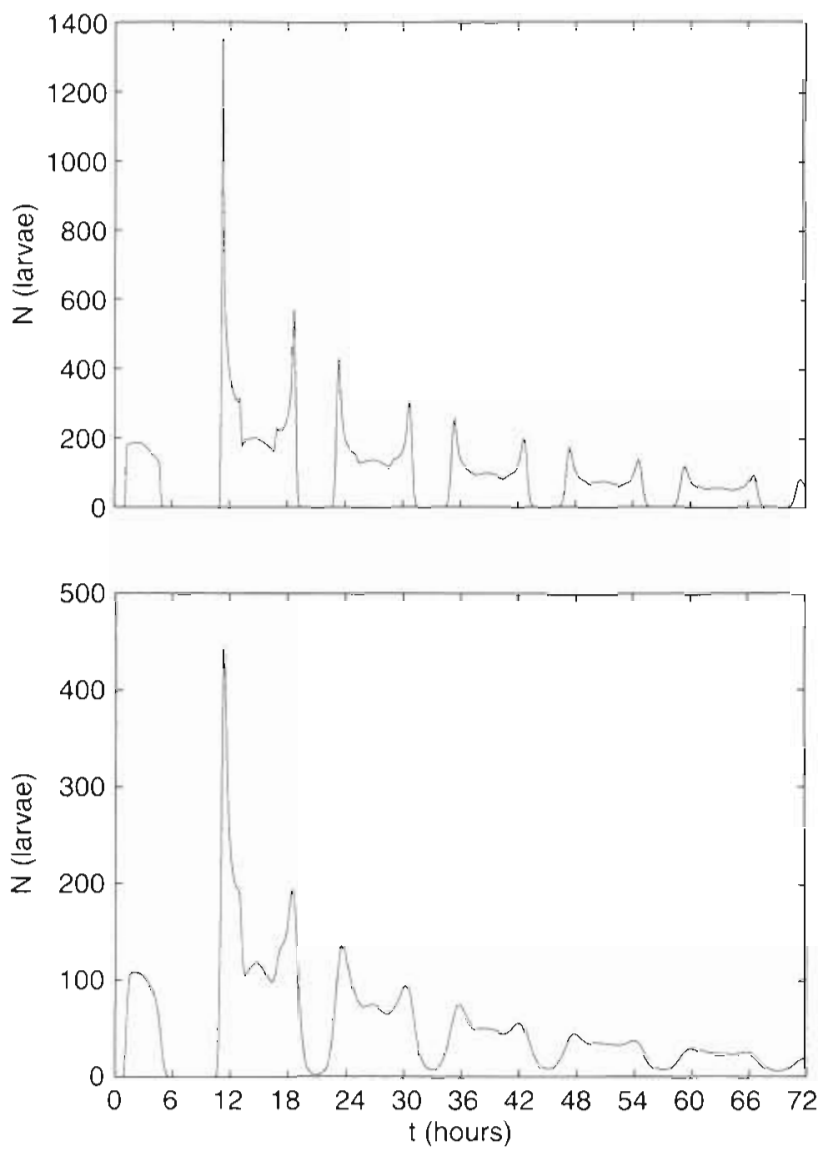

Fig. 5. Number of larvae, $N(t)$, at the downstream settlement site for a $12 \mathrm{~h}$ release into the current given by Eq. (6) with diffusion rates (a) $K_{x x}=10000 \mathrm{~m}^{2} \mathrm{~h}^{-1}, K_{y y}=1000 \mathrm{~m}^{2} \mathrm{~h}^{-1}$; and (b) $K_{x x}=100000 \mathrm{~m}^{2} \mathrm{~h}^{-1}, K_{y y}=10000 \mathrm{~m}^{2} \mathrm{~h}^{-1}$

newly released larvae. From Fig. 4 we can see why the first and second occurrences of the larval cloud at the settlement site are quite different in shape. The 2 dominant spikes present in the second and subsequent passes of the cloud are due to the high concentration of larvae produced during slack water at the 9th hour. This peak in concentration periodically moves back and forth past the settlement site gradually decreasing in magnitude due to larval mortality and the processes of diffusion. The graph given in Fig. 4 is used to explain the shape of the more complicated curves given in Fig. 5 and hence is useful in qualitatively verifying the analytic solution.

Table 1. Diffusion parameters used in the model

\begin{tabular}{|lrc|}
\hline Case & $K_{x x}\left(\mathrm{~m}^{2} \mathrm{~h}^{-1}\right)$ & $K_{y y}\left(\mathrm{~m}^{2} \mathrm{~h}^{-1}\right)$ \\
\hline Low rates of diffusion & 10000 & 1000 \\
High rates of diffusion & 100000 & 10000 \\
\hline
\end{tabular}


Contour plots of larval distribution, $S(x, y, t), 36 \mathrm{~h}$ after the start of release are given in Fig. 6 for both cases of eddy diffusion. Here we can see the effects of diffusion in spreading the larvae offshore. An increase in the rate of diffusion in the $y$-direction has resulted in larvae being mixed further offshore, leaving fewer numbers of larvae within reach of the settlement site. Thus the rate of cross-shore mixing appears to be an important factor in determining the number of competent larvae that will encounter a coastal settlement site. If the rate of mixing is low, then larvae tend to always remain close to the coast whereas if the rate is increased larvae are more likely to drift offshore away from desired settlement sites and hence eventually die from mortality effects. The contour plots also show the 2 peaks in concentration within each cloud. In both cases as time progresses the 2 peaks in concentration continue to diffuse and eventually merge to form a single peak. Again it is stressed that the model provides a statistical average of the number of larvae and hence the shape and internal structure of the larval cloud would in reality be more complicated than is suggested by these smooth contour plots.
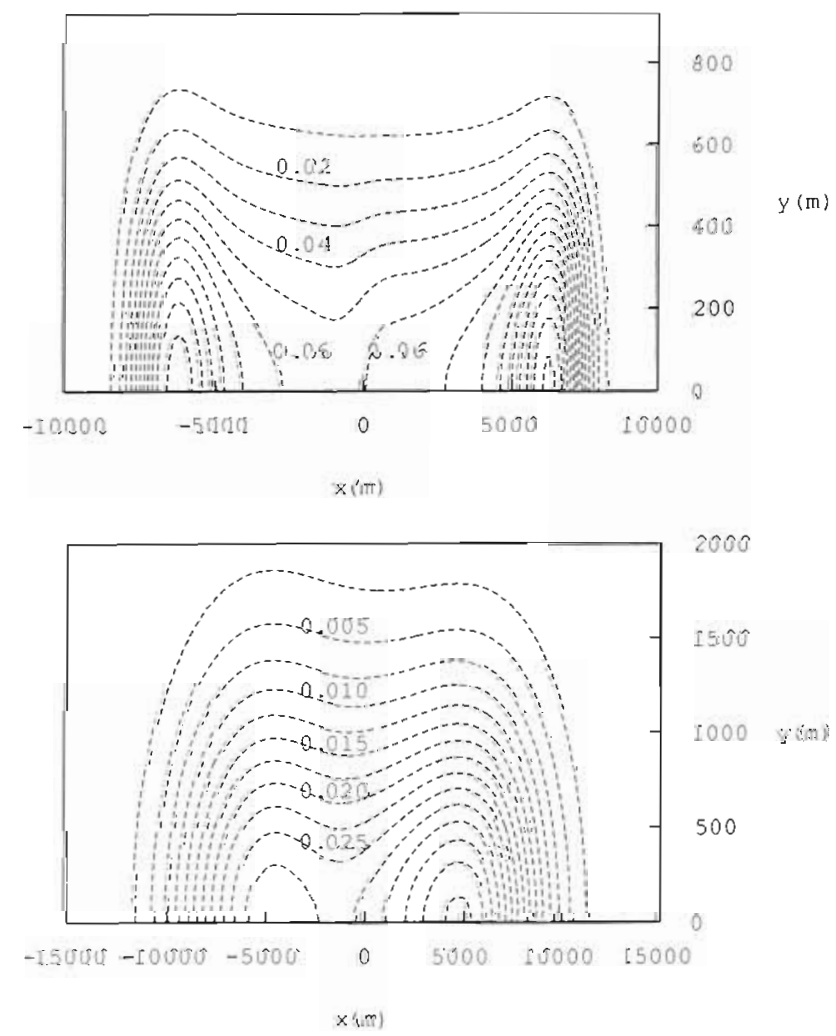

Fig. 6. Contour plots of the depth integrated concentration, $S(x, y, t), 36 \mathrm{~h}$ after the start of release. The release is continuous over a period of $12 \mathrm{~h}$ into the current given by Eq. (6) with diffusion rates (a) $K_{x x}=10000 \mathrm{~m}^{2} \mathrm{~h}^{-1}, K_{y y}=1000 \mathrm{~m}^{2} \mathrm{~h}^{-1}$; and (b) $K_{x x}=100000 \mathrm{~m}^{2} \mathrm{~h}^{-1}, K_{y y}=10000 \mathrm{~m}^{2} \mathrm{~h}^{-1}$

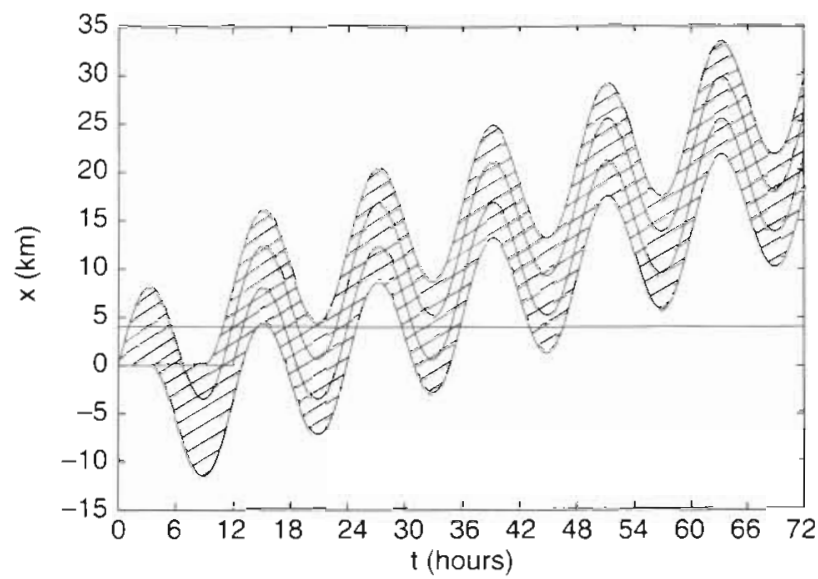

Fig. 7 Approximate along-shore distribution of larvae when released continuously for $12 \mathrm{~h}$ into the current regime given by Eq. (7)

The model is now driven with the current given by Eq. (7) to see the effect the constant along-shore component, $u_{0}$, has on the patterns of dispersal. We consider the same release conditions as before and again ruin the mouled with the 2 cases of eddy diffusion givern in Table 1.

We can approximate the length and location of the larval cloud from the current profile as before. This approximation is given in Fig. 7. As expected the addition of the along-shore component has removed the symmetry of the cloud movements about the release site. Hence the periodic appearance of roughly symmetric patterns of larval numbers drifting over the settlement site will be replaced by a more complicated pattern. The larval cloud now drifts with a mean speed of $360 \mathrm{~m}$ $\mathrm{h}^{-1}$ downstream and so the times when larvae are present at the settlement site becomes limited. We can see that in the absence of diffusion effects larvae must be prepared to settle within less than $48 \mathrm{~h}$ from the start of release if they are to have any chance of being recruited to the downstream site. Also, Fig. 7 shows that 2 slack conditions in the current occur during the release period ( $t \approx 3.2$ and $8.8 \mathrm{~h}$ ) and as a result 2 peaks in larval concentration will form as before.

Graphs of the analytic solution for the number of larvae, $N(t)$, at the downstream settlement site for both pairs of diffusion rates are given in Fig. 8. Here we see a dramatic change in the patterns of larval drift over the settlement site when compared with the previous current. Contour plots of the cloud show a similar shape and size as before with corresponding rates of diffusion. The differences between the numbers observed at the downstream site is because unlike previously the same section of the cloud is not passing the settlement site with a constant period. This removes any repeating patterns being observed, resulting in irregular numbers of larvae drifting over the settle- 

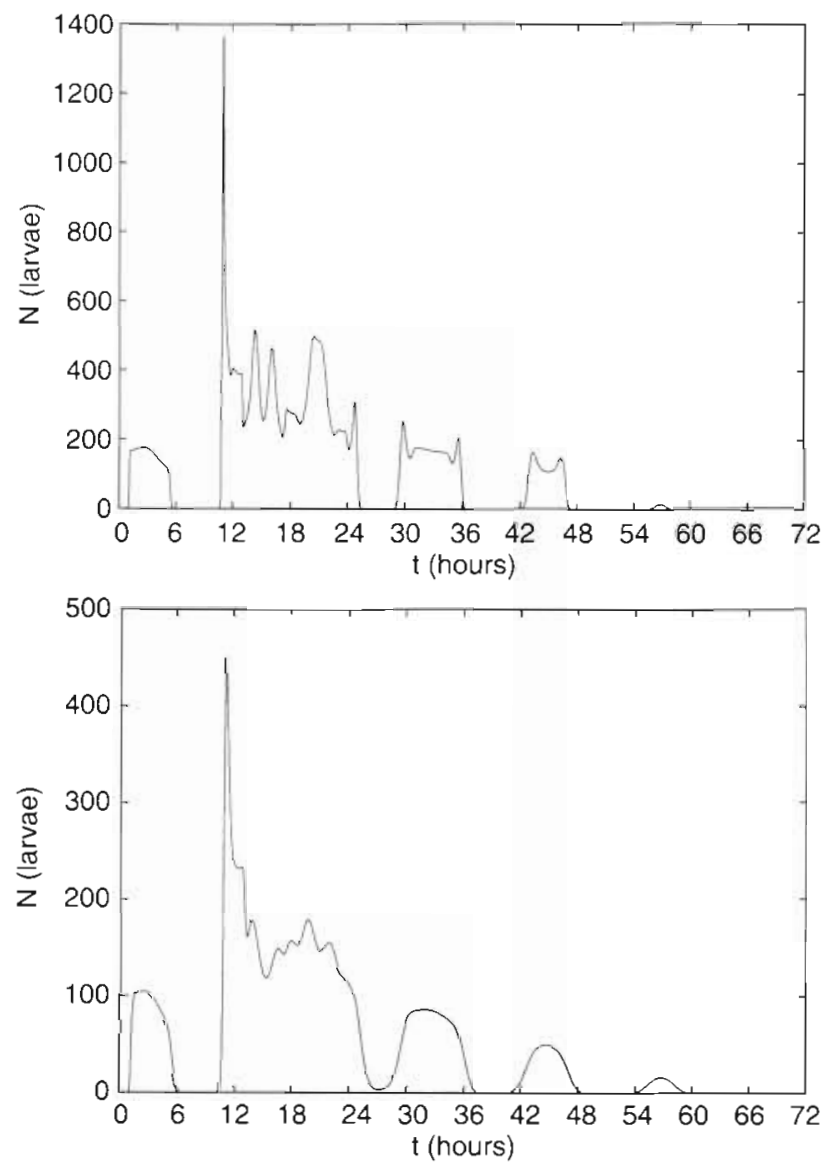

Fig. 8. Number of larvae, $N(t)$, at the downstream settlement site for a $12 \mathrm{~h}$ release into the current given by Eq. (7) with diffusion rates (a) $K_{x x}=10000 \mathrm{~m}^{2} \mathrm{~h}^{-1}, K_{y y}=1000 \mathrm{~m}^{2} \mathrm{~h}^{-1}$; and (b) $K_{x x}=100000 \mathrm{~m}^{2} \mathrm{~h}^{-1}, K_{Y y}=10000 \mathrm{~m}^{2} \mathrm{~h}^{-1}$

ment site with time. As expected the higher rates of diffusion have moved more of the larvae offshore and smoothed the fluctuations in numbers observed at the settlement site. In fact, after $30 \mathrm{~h}$ the larval cloud in the more turbulent water has lost most of its irregularities to diffusion and as a result the numbers observed when the cloud passes are very smooth unlike the less turbulent case. Fig. 8 also shows the periods in time when larvae are present over the settlement site. The increase in turbulence has increased the size of the cloud and as a result the periods when larvae are present at the settlement site become longer.

If the duration of spawning is increased from $12 \mathrm{~h}$ for the current regime described by Eq. (6), then from Fig. 4 we can see that the larvae released after the 12 th hour are always mixed with earlier released larvae. For example, 1 of the 2 peaks in concentration within the cloud is always present at the release site during a slack (i.e. 15th hour, 21st hour, etc.) and so the newly formed peaks in concentration are superimposed over the old. Thus even if the duration of spawn- ing is longer than $12 \mathrm{~h}$ the general shape and size of the cloud remains and hence the patterns of larval numbers present at the settlement sites is also similar. This is not the case when the current regime of Eq. (7) is considered. Now, peaks in concentration formed during slack periods are not superimposed over previously formed peaks and as a result the structure of the larval cloud can become extremely complicated.

Fig. 9 shows larval numbers, $N(t)$, at the downstream site when 1000000 larvae are released at a constant rate over a period of $48 \mathrm{~h}$ ( 4 tidal cycles) when both pairs of diffusion rates given in Table 1 are considered. The rate of release can be calculated using Eq. (4) with $r(t)=1 / 48$ to give $\phi \approx 10$ larvae $\mathrm{m}^{-2} \mathrm{~h}^{-1}$, i.e. a quarter of the previous rate. From Fig. 9 we can see that for both cases the number of larvae observed fluctuates wildly. A close examination of the graphs reveals a $12 \mathrm{~h}$ cycle in numbers occurring between the 12th and 60th hours. The duration when a cycle can be observed is the same length as the duration of release and the cycle has the same period as the tide.
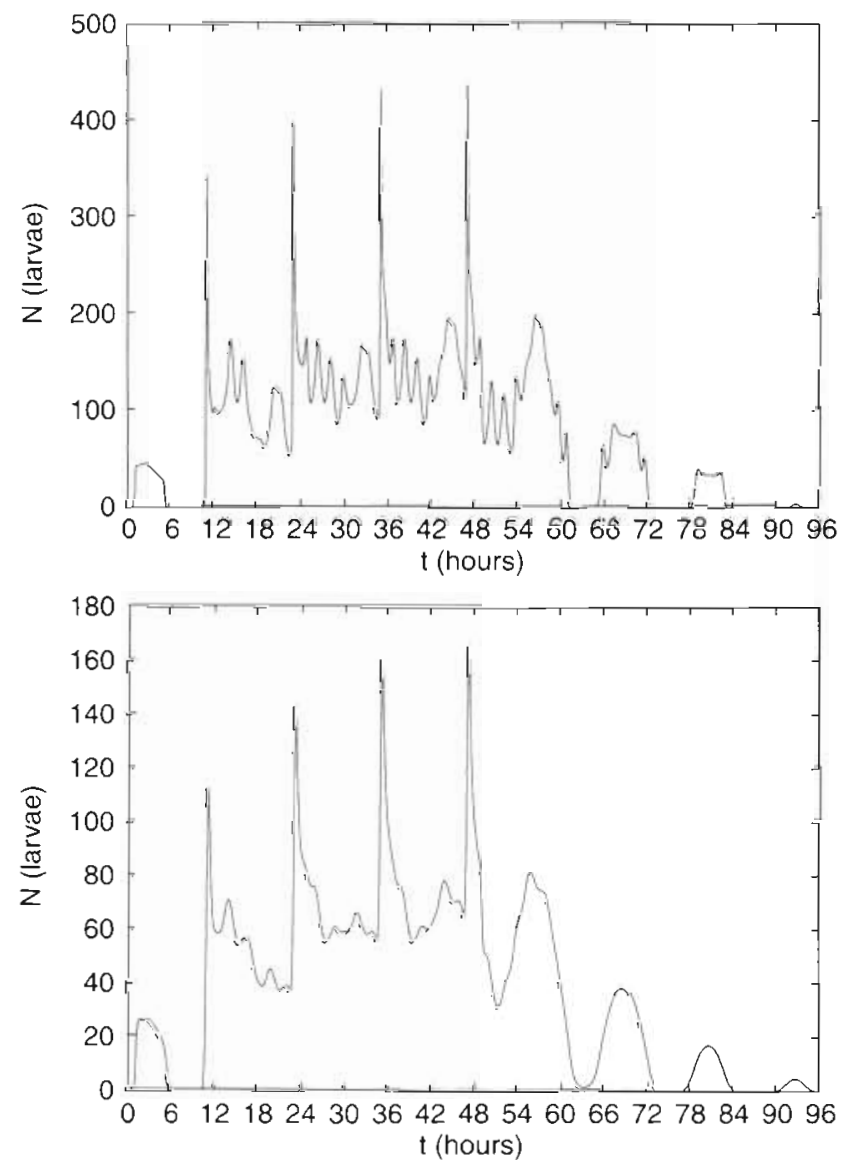

Fig. 9. Number of larvae, $N(t)$, at the downstream settlement site for a $48 \mathrm{~h}$ release into the current given by Eq. (7) with diffusion rates (a) $K_{x x}=10000 \mathrm{~m}^{2} \mathrm{~h}^{-1}, K_{y y}=1000 \mathrm{~m}^{2} \mathrm{~h}^{-1}$; and (b) $K_{x x}=100000 \mathrm{~m}^{2} \mathrm{~h}^{-1}, K_{y y}=10000 \mathrm{~m}^{2} \mathrm{~h}^{-1}$ 


\section{DISCUSSION}

In this section we briefly give the motivation for this work, explain the implications of our results and then describe some avenues for future work.

Success or failure at the pelagic larval stage of development of many marine invertebrates and vertebrates is a major determinant of the dynamics of the adult population. Thus it is important that we have a complete understanding of the processes that affect larval distribution. As field experiments have so far been unable to provide a complete picture for dispersal, a mathematical model has been constructed. We model the release of larvae over a period of time from a well defined region into an oscillating tidal current. The cloud of larvae that is produced is then tracked as it drifts along the coastline over a period of a few days. The model is also used to predict the number of larvae within d bounded region of interest, e.g. a reef or jetty.

The results from the model come from an analytic solution to the advection-diffusion-mortality equation. In order to derive an analytic solution we need to make a number of assumptions. The main assumptions are now briefly reiterated. Larvae are treated as passive particles, i.e. their movements are totally dependent on the surrounding water current. The model domain is a straight coastline with an oscillating tidal current that runs parallel to the coast. Parameters such as currents, rate of turbulent diffusion, and mortality are time dependent only, i.e. they are spatially homogeneous. The release site and settlement site are rectangular in shape. After analysing the results of the model we conclude the following

The contour plots indicate that the size and shape of the larval cloud is influenced by eddies. The larger and stronger the eddies are, the greater the mixing action within the cloud and subsequently the larger the cloud becomes. Cross-shore movement of larvae is solely due to turbulence. With the reflective boundary condition at the coast an increase in the cross-shore rate of diffusion, $K_{y y}$ results in a larger proportion of the larval cloud extending further out from the coastal waters. If settlement sites are located near the coast, then fewer larvae have the opportunity to settle (Jackson \& Strathmann 1981, Possingham \& Roughgarden 1990 ). Increasing the rate of along-shore diffusion, $K_{x x}$ lengthens the cloud which in turn increases the time frame when larvae are present at a coastal site. This effect can be seen in Figs. 5 \& 8.

The model indicates that the current, $U(t)$, plays an important role in controlling where the larvae drift and hence where settlement can take place. The interaction of the current with the timing and duration of spawning determines the initial size and concentration profile of the larval cloud. Larvae that are released from a site into a fast-moving stream of water are spread more quickly over a greater area than if the larvae are released into slack water. Thus the density of larvae within the cloud is dependent on the current strength at the time of release.

The timing of larval release with the phase of the tide is important in determining the length of the cloud especially when the duration of release is of the order of less than a tidal period. For example, release over a short period of time into a fast moving current will produce a larval cloud which is larger than if release is during slack water. The larger the cloud produced during release, the longer the extent of coastline becomes where settlement may take place. For a current with no along-shore component there is little to be gained in terms of increasing the length of the cloud by releasing larvae over a period longer than the tide (e.g. $12 \mathrm{~h}$ in this case). However, if the current does have an alongshore component, then the ionger the larvae are released the longer the larval cloud becomes.

The length of the precompetent period and the competent period along with the flow regime are important in determining when and where settlement can take place. For a flow with no along-shore component such as the one given by Eq. (6) settlement at the release site is possible regardless of the time that larvae must spend in the water developing. For a current with an along-shore component, the time frame when larvae are capable of settling is crucial in determining where along the coastline settlement may take place. For the current given by Eq. (7) if the precompetent period is longer than $40 \mathrm{~h}$, unless turbulent diffusion effects are large it would appear unlikely that larvae would return to the release site in a state ready to settle. Thus new recruits to the release site must come from other spawning grounds located upstream. If this is the case, then an unsuccessful spawning at a release site due to changes in the environment will effect the dynamics of other populations located downstream. For species with a long precompetent period the success of one population may be dependent on the success of neighbouring populations.

From the model presented we can see that the scale of dispersal is dependent on the interaction of many processes. These include current profiles, turbulence effects, mortality effects, the length of the precompetent and competent period, spawning characteristics and size and location of settlement sites. Hence there is the possibility for great variability in the range of successful settlement if any of these processes are disturbed or altered in any way.

Even with the simple assumptions, the model has produced surprisingly complex patterns of dispersal and relaxing assumptions could only introduce more complexity. Thus the testing of more complex models 
of larval dispersal is likely to be impossible as the effects of changing parameter values and the forms of functions may be difficult to detect. Another implication of the model is in the analysis of field data. The model has shown that what may at a first glance appear to be chaotic fluctuations in larvae numbers is not necessarily the result of a complex current or larval behaviour. Thus it may be futile to attempt to reconstruct such things as current structure from field data on larval densities.

The work presented here is based on dispersal over a relatively short range and period of time. As a consequence, the results and implications given here may not be applicable to larger scale dispersal. We expect that for larvae that are released from much larger regions and spend longer periods in the pelagic then smaller scale processes such as the half daily oscillatory component of the tide, and the duration and rate of release will play a lesser role in dispersal.

Many extensions can be made to the model but as stated above their effect on the results will need to carefully examined. Below some of the extensions are discussed.

By using the depth-averaged ADME we have had to ignore vertical effects in many of the parameters. For example the current, $U(t)$, is a depth-averaged velocity and in reality it may vary considerably in direction and speed from the surface to the seabed. We have assumed that larvae are neutrally buoyant and are uniformly distributed throughout a given section of the water column but if larvae undergo daily vertical migration as has been reported for some species of larvae (Richter 1973, Barnes \& Mann 1991, Boudrea et al. 1991. Hill 1991b) then movements may be different to that presented here. In fact dispersal may be controlled to some extent by larvae altering their vertical position such that they always reside in water that flows in a certain direction (Hofmann 1993). By including the vertical we could more accurately model the shear effect on larvae.

Spatial homogeneity is assumed in many of the variables in order to allow for an analytic solution. These variables include the current, mortality, rate of spawning and turbulence effects. In reality we would expect these parameters to vary in magnitude with spatial position. To include spatial dependence will require the model equations to be solved by purely numerical techniques such as the method of finite elements or finite differences.

Here we have assumed a geometrically simple coastline and a simple flow. The next step is to consider an actual coastline with real currents. This involves running a numerical tidal model over the region and extracting the required velocity vectors for input to the larval model (Noye et al. 1992). It has been suggested that the geometry of the coast and the sea bed and wind stress may be important in generating secondary currents that tend to aggregate larvae into slicks (Shanks \& Wright 1987. Wolanski \& Hamner 1988). These secondary currents may be in the form of Langmuir circulations or internal waves. Thus a good model of dispersal should be able to resolve such secondary circulations and the role they play in larval aggregation.

Acknowledgements. We thank the numerical and ecological modelling groups in the Applied Mathematics Department for their suggestions and support, and the anonymous referees for their comments. Financial assistance from an Australian Postgraduate Research Award (Priority) is gratefully acknowledged.

\section{Appendix}

In this appendix we give the semi-analytic solutions for the depth-integrated larval concentration, $S(x, y, t)$, and the number of larvae at the potential settlement site, $N(t)$. We use the term semi-analytic as the solutions are expressed in the form of an integral equation which itself cannot be solved for analytically but can be accurately approximated by numerical integration techniques.

First we consider Eq. (2) when it is subject to the reflective boundary condition at the coast. The solution for the depthintegrated larval concentration is given by

$$
S(x, y, t)=\int_{0}^{t m} \frac{\phi(\tau)}{4} \exp \{-\bar{M}(t, \tau)\} G(x, t, \tau) H(y, t, \tau) \mathrm{d} \tau
$$

where

$$
\begin{aligned}
& t_{m}=\min (t, T) \\
& \phi(\tau)=\frac{L \Gamma(\tau)}{4 A_{R} B_{R}} \\
& \bar{M}(t, \tau)=\int_{\tau}^{t} M\left(\tau^{\prime}\right) d \tau^{\cdot} \\
& G(x, t, \tau)=\operatorname{erf}\left\{\alpha_{2}(x, t, \tau)\right\}-\operatorname{erf}\left\{\alpha_{1}(x, t, \tau)\right\} \\
& H(y, t, \tau)=\operatorname{erf}\left\{\beta_{2}(y, t, \tau)\right\}-\operatorname{erf}\left\{\beta_{1}(y, t, \tau)\right\} \\
& \quad+\operatorname{erf}\left\{\bar{\beta}_{2}(y, t, \tau)\right\}-\operatorname{erf}\left\{\bar{\beta}_{1}(y, t, \tau)\right\} \\
& \alpha_{1}(x, t, \tau)=\frac{X(x, t, \tau)-x_{R}-A_{R}}{2 \sqrt{T_{x}}(t, \tau)}
\end{aligned}
$$


Appendix (continued)

$$
\begin{aligned}
& \alpha_{2}(x, t, \tau)=\frac{X(x, t, \tau)-x_{R}+A_{R}}{2 \sqrt{T_{x}(t, \tau)}} \\
& \beta_{1}(y, t, \tau)=\frac{y-y_{R}-B_{R}}{2 \sqrt{T_{y}(t, \tau)}}- \\
& \beta_{2}(y, t, \tau)=\frac{y-y_{R}+B_{R}}{2 \sqrt{T_{y}(t, \tau)}} \\
& \bar{\beta}_{1}(y, t, \tau)=\frac{y+y_{R}-B_{R}}{2 \sqrt{T_{y}(t, \tau)}} \\
& \bar{\beta}_{2}(y, t, \tau)=\frac{y+y_{R}+B_{R}}{2 \sqrt{T_{y}(t, \tau)}} \\
& X(x, t, \tau)=x-\int_{\tau}^{t} U\left(\tau^{\circ}\right) \mathrm{d} \tau^{\circ} \\
& T_{x}(t, \tau)=\int_{\tau}^{t} K_{x x}\left(\tau^{\circ}\right) \mathrm{d} \tau^{\circ} \\
& T_{y}(t, \tau)=\int_{\tau}^{t} K_{y y}\left(\tau^{\prime}\right) \mathrm{d} \tau^{\circ}
\end{aligned}
$$

and erf $(x)$ is the error function defined by the integral

$$
\operatorname{erf}(x)=\frac{2}{\sqrt{\pi}} \int_{0}^{x} \exp \left(-w^{2}\right) \mathrm{d} w
$$

Due to the absence of experimental data, for all the results given in this paper we assumed time homogeneous functional forms for the fractional rate of larval release, $r(t)$ larval mortality, $M(t)$, and the transverse rates of turbulent diffusion, $K_{x x}(t)$ and $K_{y y}(t)$. This leads to the following simplifications,

$$
\begin{aligned}
& \phi(\tau)=\frac{L}{4 T A_{R} B_{R}} \\
& \bar{M}(t, \tau)=(t-\tau) M \\
& T_{x}(t, \tau)=(t-\tau) K_{x x} \\
& T_{y}(t, \tau)=(t-\tau) K_{y \gamma}
\end{aligned}
$$

To obtain the solution to $N(t)$, we integrate the above solution for $S(t)$ over the region of the settlement site. It can be shown that the solution to this integral is given by

$$
N(t)=\int_{0}^{l_{m}} \frac{\phi(\tau)}{4} \exp \{-\bar{M}(t, \tau)\} \hat{G}(t, \tau) \hat{H}(t, \tau) \mathrm{d} \tau
$$

where

$$
\begin{aligned}
\hat{G}(t, \tau)= & I\left\{\alpha_{2}\left(x_{S}+A_{S}, t, \tau\right)\right\}-I\left\{\alpha_{2}\left(x_{S}-A_{S}, t, \tau\right)\right\} \\
& -I\left\{\alpha_{1}\left(x_{S}+A_{S}, t, \tau\right)\right\}+I\left\{\alpha_{1}\left(x_{S}-A_{S}, t, \tau\right)\right\} \\
\hat{H}(t, \tau)= & I\left\{\beta_{2}\left(y_{S}+B_{S}, t, \tau\right)\right\}-I\left\{\beta_{2}\left(y_{S}-B_{S}, t, \tau\right)\right\} \\
& -I\left\{\beta_{1}\left(y_{S}+B_{S}, t, \tau\right)\right\}+I\left\{\beta_{1}\left(y_{S}-B_{S}, t, \tau\right)\right\} \\
& +I\left\{\bar{\beta}_{2}\left(y_{S}+B_{S}, t, \tau\right)\right\}-I\left\{\bar{\beta}_{2}\left(y_{S}-B_{S}, t, \tau\right)\right\} \\
& -I\left\{\bar{\beta}_{1}\left(y_{S}+B_{S}, t, \tau\right)\right\}+I\left\{\bar{\beta}_{1}\left(y_{S}-B_{S}, t, \tau\right)\right\} \\
I(z)= & z \operatorname{erf}(z)+\frac{1}{\sqrt{\pi}}\left[\exp \left(-z^{2}\right)-1\right]
\end{aligned}
$$

\section{LITERATURE CITED}

Barnes RSK, Mann KH (1991) Fundamentals of aquatic ecology, 2nd edn. Blackwell Scientific Publications, Oxford

Boudrea B, Simard Y, Bourget E (1991) Behavioural responses of the planktonic stages of the American lobster Homarus americanus to thermal gradients and ecological implications. Mar Ecol Prog Ser 76:13-23

Bowden KF (1964) Turbulence. Oceanogr mar Biol A Rev 2: $11-30$

Butler AJ (1987) Ecology of Pinna bicolor gmelin (Mollusca: Bivalvia) in Gulf St. Vincent, South Australia: density, reproductive cycle, recruitment, growth and mortality at three sites. Aust J mar Freshwat Res 38:743-769

Butman CA (1987) Larval settlement in soft-sediment invertebrates: the spatial scales of pattern explained by active habitat selection and the emerging role of hydrodynamical processes. Oceanogr mar Biol A Rev 25:113-165

Conte SD, de Boor C (1988) Elementary numerical analysis, an algorithmic approach, 3rd edn. McGraw-Hill Book Company, Singapore

De Wolf P (1973) Ecological observations on the mechanisms of dispersal of barnacle larvae during planktonic life and settling. Neth J Sea Res 6:1-129

Dight IJ, Bode L, James MK (1990) Modelling the larval dispersal of Acanthaster planci, I. Large scale hydrodynamics, Cairns Section, Great Barrier Reef Marine Park. Coral Reefs 9:115-123

Fairweather PG (1991) Implications of 'supply-side' ecology for environmental assessment and management. Trends Ecol Evol 6:60-63
Fischer HB, List EJ, Koh RCY, Imberger J, Brooks NH (1979) Mixing in inland and coastal waters. Academic Press, New York

Gaines SD, Bertress MD (1992) Dispersal of juveniles and variable recruitment in sessile marine species. Nature 360 $579-580$

Hannan CA (1984) Planktonic larvae may act like passive particles in turbulent near-bottom flows. Limnol Oceanogr 29 $1108-1116$

Havenhand JN (1991a) Fertilisation and the potential for dispersal of gametes and larvae in the solitary ascidian Ascidia mentula muller. Ophelia 33:1-15

Havenhand JN (1991b) On the behaviour of opisthobranch larvae. J Moll Stud 57:119-131

Hill AE (1990) Pelagic dispersal of Norway lobster Nephrops norvegicus larvae examined using an advection-diffusionmortality model. Mar Ecol Prog Ser 64:217-226

Hill AE (1991a) Advection-diffusion-mortality solutions for investigating pelagic larval dispersal. Mar Ecol Prog Ser $70: 117-128$

Hill AE (1991b) Vertical migration in tidal currents. Mar Ecol Prog Ser 75:39-54

Hinckley S, Bailey $K$, Picquelle S, Yoklavich $M$, Stabeno P (1993) Age-specific mortality and transport of larval walleye pollock Theragra chalcogramma in the western Gulf of Alaska. Mar Ecol Prog Ser 98:17-29

Hofmann EE (1993) Coupling of circulation and marine ecosystem models. In: Levin SA, Powell TM, Steele JH (eds) Patch dynamics. Lecture notes in biomathematics, Vol 96. Springer-Verlag. Berlin, p 136-158

Holly FM, Usseglio JM (1984) Dispersal simulation in two- 
dimensional tidal flow. J Hydr Eng 110:905-926

Jackson GA, Strathmann RR (1981) Larval mortality from offshore mixing as a link between precompetent and competent periods of development. Am Nat 118:16-26

James MK, Scandol JP (1992) Larval dispersal simulations: correlations with the Crown-of-thorns starfish outbreaks database. Aust J mar Freshwat Res 43:569-582

Johnson DF, Hess KW (1990) Numerical simulations of blue crab larval dispersal and recruitment. Bull mar Sci 46:195-213

Koehl MAR, Powell TM, Dairiki G (1993) Measuring the fate of patches in the water: larval dispersal. In: Levin SA, Powell TM, Steele JH (eds) Patch dynamics. Lecture notes in biomathematics, Vol 96. Springer-Verlag, Berlin, $p$ $50-60$

Mathews JH (1987) Numerical methods for computer science, engineering, and mathematics. Prentice-Hall, Englewood Cliffs, NJ

McGurk MD (1989) Advection, diffusion and mortality of Pacific herring larvae Clupea harengus pallasi in Bamfield Inlet, British Columbia. Mar Ecol Prog Ser 51:1-18

Menge BA. Sutherland JP (1987) Community regulation: variation in disturbance, competition, and predation in relation to environmental stress and recruitment. Am Nat 130:730-757

Minchinton TE, Scheibling RE (1991) The influence of larval supply and settlement on the population structure of barnacles. Ecology 72:1867-1879

Noye BJ (1984) Physical processes and pollution in the water of Spencer Gulf. Mar Geol 61:197-220

Noye BJ, Bills PJ, I'Anson K, Wong CC (1992) Prediction of prawn larvae movement in a coastal sea. In: Noye BJ, Benjamin BR, Colgan LH (eds) Computational Techniques and Applications Conference: CTAC-91. Computational Mathematics Group, Australian Mathematical Society, Adelaide, p 385-392

Okubo A (1971) Oceanic diffusion diagrams. Deep Sea Res 18:789-802

Okubo A (1976) Remarks on the use of 'diffusion diagrams' in modelling scale-dependent diffusion. Deep Sea Res 23: $1213-1214$

Okubo A (1980) Diffusion and ecological problems: mathe-

This article was submitted to the editor matical models, Biomathematics, Vol 10. Springer-Verlag, Berlin

Planes S (1993) Genetic differentiation in relation to restricted larval dispersal of the convict surgeonfish Acanthurus triostegus in French Polynesia. Mar Ecol Prog Ser 98: $237-246$

Possingham HP, Roughgarden J (1990) Spatial population dynamics of a marine oganism with a complex life cycle. Ecology 71:973-985

Power JH (1984) Advection, diffusion, and drift migrations of larval fish. Mechanisms of migration in fishes. Plenum Press, New York, p 27-37

Richter G (1973) Field and laboratory observations on the diumal vertical migration of marine gastropod larvae. Neth J Sea Res 7:126-134

Roughgarden J, Gaines S, Possingham H (1988) Recruitment dynamics in complex life cycles. Science 241:1460-1466

Sale PF (1990) Recruitment of marine species: is the bandwagon rolling in the right direction? Trends Ecol Evol 5: $25-27$

Scandol JP, James MK (1992) Hydrodynamics and larval dispersal: a population model of Acanthaster planci on the Great Barrier Reef. Aust J mar Freshwat Res 43:583-596

Shanks AL, Wright WG (1987) Internal-wave-mediated shoreward transport of cyprids, megalope, and gammarids and correlated longshore differences in the settling rate of intertidal barnacles. J exp mar Biol Ecol 114:1-13

Shepherd SA, Lowe D, Partington D (1992) Studies on southern Australian abalone (Genus Haliotis) XIII. Larval dispersal and recruitment. J exp mar Biol Ecol 164:247-260

Strathmann R (1974) The spread of sibling larvae of sedentary marine invertebrates. Am Nat 108:29-44

Todd CD, Havenhand JN, Thorpe JP (1988) Genetic differentiation, pelagic larval transport and gene flow between local populations of the intertidal marine mollusc Adalaria proxima (Alder \& Hancock). Funct Ecol 2:441-451

Walters LJ (1992) Field settlement locations on subtidal marine hard substrata: is active larval exploration involved? Limnol Oceanogr 37:1101-1107

Wolanski E, Hamner WM (1988) Topographically controlled fronts in the ocean and their biological influence. Science $241: 177-181$

Manuscript first received: October 11, 1994

Revised version accepted: February 16, 1995 